\title{
Hairy magnetic and dyonic black holes in the Standard Model
}

\author{
Yang Bai and Mrunal Korwar \\ Department of Physics, University of Wisconsin-Madison, \\ Madison, WI 53706, U.S.A. \\ E-mail: yangbai@physics.wisc.edu, mkorwar@wisc.edu
}

ABSTRACT: Spherically symmetric magnetic and dyonic black holes with a magnetic charge $Q=2$ are studied in the Standard Model and general relativity. A magnetically charged black hole with mass below $9.3 \times 10^{35} \mathrm{GeV}$ has a "hairy" cloud of electroweak gauge and Higgs fields outside the event horizon with $1 / m_{W}$ in size. An extremal magnetic black hole has a hair mass of $3.6 \mathrm{TeV}$, while an extremal dyonic black hole has an additional mass of $q^{2} \times 1.6 \mathrm{GeV}$ for a small electric charge $q \ll 2 \pi / e^{2}$. A hairy dyonic black hole with an integer charge is not stable and can decay into a magnetic one plus charged fermions. On the other hand, a hairy magnetic black hole can evolve via Hawking radiation into a nearly extremal one that is cosmologically stable and an interesting object to be searched for.

KEYwords: Black Holes, Beyond Standard Model

ArXiv EPrint: 2012.15430 


\section{Contents}

1 Introduction 1

2 Lagrangian and equations of motion 2

3 Hairy magnetic black holes 4

3.1 Masses and profiles 4

3.2 Hawking temperature and black hole evaporation 8

4 Hairy dyonic black holes $\quad 10$

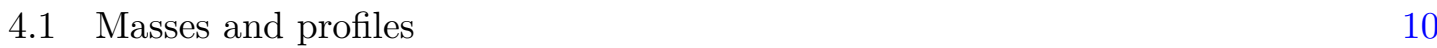

$\begin{array}{lll}4.2 & \text { Some properties of hairy dyonic black holes } & 14\end{array}$

5 Discussion and conclusions $\quad 15$

\section{Introduction}

Although the black hole no-hair conjecture has been proposed a long time ago [1], various violations have been found in the systems like Einstein-Skyrme [2-5] and Einstein-YangMills-Higgs [6-12] (see [13] for a review). For instance, refs. [6, 7] have demonstrated that the magnetically charged Reissner-Nordström $(\mathrm{RN})$ black holes is classically unstable and can evolve into a hairy magnetic black hole with a cloud of gauge and Higgs field configures. The existence of a non-trivial hair for the black hole changes the black hole properties in various ways including the faster and effectively two-dimensional Hawking radiation [14]. Given the discovery of the Higgs boson in $2012[15,16]$ and the completeness of the Standard Model (SM), we study the properties of magnetic and dyonic black holes with electroweak gauge and Higgs hair. In our study, we will consider the magnetic charge $Q=2$ case with a spherically symmetric configuration for the whole system. We want to emphasize that the existence of hairy magnetic and dyonic black holes only depends on the known SM and general relativity, although their abundance in the current Universe is subject to the early-universe formation mechanism and various phenomenological constraints [17].

In the direction of searching for localized and magnetically charged object with finite energy, there is no viable candidate just based on the SM interactions (see [18, 19] for studies including physics beyond the SM). The existence of a black hole inside a magnetic monopole can make the energy of the whole system finite, because the divergent behavior of the monopole energy in the small radius region is regularized by the black hole event horizon. So, the hairy magnetic black hole (hMBH) serves as a unique and justified magnetically charged object based on experimentally established SM (on the purely theoretically side, we may have the t 'hooft-Polyakov monopole [20, 21] in the grand unified theories [22]). 
This paper is organized as follows. We first derive the equations of motion for a general hairy dyonic black hole $(\mathrm{hDBH})$ in section 2. In section 3.1, we solve the equations of motion with proper boundary conditions (BCs) and calculate the mass and the field profiles for the hairy magnetic black hole case. We discuss the Hawking temperature and evolution of non-extremal hairy magnetic black hole in section 3.2. In section 4.1, the mass and field profiles for the hairy dyonic black holes will be calculated with some of their properties discussed in section 4.2. We conclude our paper and discuss some future directions in section 5 .

\section{Lagrangian and equations of motion}

Starting with the Einstein-Hilbert action in general relativity and including the matter Lagrangian in the SM of particle physics, one has the action

$$
S=\int d^{4} x \sqrt{-g}\left[-\frac{1}{16 \pi G} R+\mathcal{L}_{\mathrm{SM}}\right] .
$$

Here, $G=1 / M_{\mathrm{pl}}^{2}$ with $M_{\mathrm{pl}}=1.22 \times 10^{19} \mathrm{GeV}$ is the Newton constant; $R$ is the Ricci scalar; $g \equiv \operatorname{det}\left(g_{\mu \nu}\right)$ is the determinant of the metric tensor matrix; the metric $g_{\mu \nu}=\left(g^{\mu \nu}\right)^{-1}$ has the convention of $(+1,-1,-1,-1)$ for a flat-space metric. For the matter Lagrangian, we only include the known SM Lagrangian with focus on the electroweak $\mathrm{SU}(2)_{W} \times \mathrm{U}(1)_{Y}$ gauge sector

$$
\mathcal{L}_{\mathrm{SM}} \supset \mathcal{L}_{\mathrm{EW}}=-\frac{1}{4} W_{\mu \nu}^{a} W^{a \mu \nu}-\frac{1}{4} Y_{\mu \nu} Y^{\mu \nu}+\left|D_{\mu} H\right|^{2}-\frac{\lambda}{2}\left(H^{\dagger} H-\frac{v^{2}}{2}\right)^{2},
$$

with $v=246 \mathrm{GeV}$ and $\lambda \approx 0.26$ to have the Higgs boson mass of $m_{h}=\sqrt{\lambda} v \approx 125 \mathrm{GeV}$ [23]. Here, $W_{\mu}^{a}$ with $a=1,2,3$ are $\mathrm{SU}(2)_{W}$ gauge bosons and $Y_{\mu}$ is the hypercharge gauge boson. The gauge field tensors are $W_{\mu \nu}^{a}=\partial_{\mu} W_{\nu}^{a}-\partial_{\nu} W_{\mu}^{a}-g \epsilon_{a b c} W_{\mu}^{b} W_{\nu}^{c}$ and $Y_{\mu \nu}=\partial_{\mu} Y_{\nu}-\partial_{\nu} Y_{\mu}$. The covariant derivative of the Higgs doublet is

$$
D_{\mu} H=\left(\partial_{\mu}-i \frac{g}{2} \sigma^{a} W_{\mu}^{a}-i \frac{g_{Y}}{2} Y_{\mu}\right) H
$$

with $\sigma^{a}$ as the Pauli matrices and the two gauge couplings $g=e / \sin \theta_{W}$ and $g_{Y}=e / \cos \theta_{W}$ with $e=\sqrt{4 \pi \alpha}$ and $\alpha \approx 1 / 128$ at the electroweak scale. Here, $\theta_{W}$ is the weak mixing angle with $\sin \theta_{W} \approx \sqrt{0.23}$. The constant term for the Higgs potential is chosen to have a zero value when the Higgs field sits at the potential minimum $\langle H\rangle=(0, v / \sqrt{2})^{T}$.

For both magnetic and dyonic black holes, we will consider only the $Q=2$ magnetic charge in this paper (we will use $Q$ to label the magnetic charge and $q$ for the electric charge). At a long distance, the magnetic field is $\mathbf{B}(\mathbf{r})=Q e_{\mathrm{M}} \hat{\mathbf{r}} /\left(4 \pi r^{2}\right)$ with the magnetic coupling $e_{\mathrm{M}}=2 \pi / e$ following the Dirac quantization for the minimum charge $Q=1$. For $Q=2$, we anticipate a spherically symmetric solution for both magnetic and dyonic black holes. Therefore, we parametrize the metric as

$$
d s^{2}=P^{2}(r) N(r) d t^{2}-N(r)^{-1} d r^{2}-r^{2} d \theta^{2}-r^{2} \sin ^{2} \theta d \phi^{2},
$$


in the spherical coordinate. For the Einstein-Hilbert action and using integrating by parts that does not change the later equations of motion, one has (see [24] for a different metric convention)

$$
S_{\mathrm{E}}=-\frac{1}{16 \pi G} \int d^{4} x \sqrt{-g} R=-\frac{1}{2 G} \int d t d r r P^{\prime}(1-N),
$$

with the prime denoting differentiation with respect to $r$.

For the matter part and following ref. [18], we use the following ansatz for a spherical monopole (or dyon) configuration in the hedgehog gauge ${ }^{1}$

$$
\begin{aligned}
H & =\frac{v}{\sqrt{2}} \rho(r) \xi, & \xi & =i\left(\begin{array}{c}
\sin \left(\frac{\theta}{2}\right) e^{-i \phi} \\
-\cos \left(\frac{\theta}{2}\right)
\end{array}\right), \\
W_{i}^{a} & =\epsilon^{a i j} \frac{r^{j}}{r^{2}}\left(\frac{1-f(r)}{g}\right), & W_{0}^{a} & =-\frac{v}{g} w(r) \frac{r^{a}}{r}, \\
Y_{i} & =-\frac{1}{g_{Y}}(1-\cos \theta) \partial_{i} \phi, & Y_{0} & =-\frac{v}{g_{Y}} y(r) .
\end{aligned}
$$

Noting that $\xi^{\dagger} \vec{\sigma} \xi=-\vec{r} / r$, so $H^{\dagger} \vec{\sigma} H$ has been treated as a triplet under $\mathrm{SU}(2)_{W}$ as the simple $\mathrm{SU}(2)$ monopole case $[20,21]$. Here, the index " $i$ " for $W_{i}^{a}$ and $Y_{i}$ is the Cartesian coordinate index. There are totally four dimensionless functions $\rho(r), f(r), w(r)$ and $y(r)$ to describe the Higgs and gauge field profiles. For the purely magnetic black hole case, one simply sets $w(r)=y(r)=0$. One can perform an $\mathrm{SU}(2)_{W}$ gauge transformation to change from the hedgehog gauge to the unitary gauge

$$
\xi \longrightarrow U \xi=\left(\begin{array}{l}
0 \\
1
\end{array}\right) \quad \text { with } \quad U=-i\left(\begin{array}{cc}
\cos \left(\frac{\theta}{2}\right) & \sin \left(\frac{\theta}{2}\right) e^{-i \phi} \\
\sin \left(\frac{\theta}{2}\right) e^{i \phi} & -\cos \left(\frac{\theta}{2}\right)
\end{array}\right) .
$$

In the unitary gauge and after rotating the neutral gauge fields from the basis $\left(Y_{\mu}, W_{\mu}^{3}\right)$ to the photon and $Z$ boson basis $\left(A_{\mu}, Z_{\mu}\right)$, one has

$$
\begin{aligned}
A_{\mu} & =-e v\left[\frac{1}{g^{2}} w(r)+\frac{1}{g_{Y}^{2}} y(r)\right] \partial_{\mu} t-\frac{1}{e}(1-\cos \theta) \partial_{\mu} \phi, \\
Z_{\mu} & =\frac{e}{g g_{Y}} v[y(r)-w(r)] \partial_{\mu} t .
\end{aligned}
$$

Note that $\partial_{0} t=1$ and $\partial_{i} t=0$. Again, for the purely magnetic black hole case with $w(r)=y(r)=0$, there is no $Z$ boson profile.

Substituting the ansatz profiles into the matter action, one has

$$
\begin{aligned}
S_{\text {matter }} & \supset \int d^{4} x \sqrt{-g} \mathcal{L}_{\mathrm{EW}} \\
& =-4 \pi \int d t d r r^{2}\left[P(r) N(r) \mathcal{K}+P(r) \mathcal{U}-P(r)^{-1} \mathcal{K}_{0}-P(r)^{-1} N(r)^{-1} \mathcal{U}_{0}\right]
\end{aligned}
$$

\footnotetext{
${ }^{1}$ The topological argument for the existence of this configure is provided in ref. [18]: $\pi_{2}\left(\mathbb{C P}^{1}\right)=\pi_{2}\left(S^{2}\right)=\mathcal{Z}$. For the Higgs doublet $H=\left(H_{1}, H_{2}\right)^{T}$ with $H_{1} H_{1}^{*}+H_{2} H_{2}^{*}=v^{2} / 2$, the vacuum manifold in the pure scalar sector is $S^{3}$ with $\pi_{2}\left(S^{3}\right)=0$. However, given the $\mathrm{U}(1)_{Y}$ gauge freedom, one could make a gauge rotation to make one of the two complex fields $H_{1,2}$ real. As a result, the manifold has a lower dimension and is isomorphic to $S^{2}$.
} 
with

$$
\begin{aligned}
\mathcal{K} & =\frac{v^{2} \rho^{\prime 2}}{2}+\frac{f^{\prime 2}}{g^{2} r^{2}}, \\
\mathcal{U} & =\frac{v^{2} f^{2} \rho^{2}}{4 r^{2}}+\frac{\left(1-f^{2}\right)^{2}}{2 g^{2} r^{4}}+\frac{\lambda}{8} v^{4}\left(\rho^{2}-1\right)^{2}+\frac{1}{2 g_{Y}^{2} r^{4}} \equiv \mathcal{U}_{1}+\frac{1}{2 g_{Y}^{2} r^{4}}, \\
\mathcal{K}_{0} & =\frac{v^{2} w^{\prime 2}}{2 g^{2}}+\frac{v^{2} y^{\prime 2}}{2 g_{Y}^{2}}, \\
\mathcal{U}_{0} & =\frac{v^{2} w^{2} f^{2}}{g^{2} r^{2}}+\frac{v^{4}(w-y)^{2} \rho^{2}}{8} .
\end{aligned}
$$

The above formulas agree with ref. [25] for the magnetic case with $w=y=0$. Note that the term $1 /\left(2 g_{Y}^{2} r^{4}\right)$ in $\mathcal{U}$ has infinite energy for the magnetic monopole without a black hole in the core $[18,19,25]$. This is another manifestation that the SM electroweak sector by itself does not admit a finite-energy magnetic monopole. Existence of a black hole event horizon can make the energy of the total system finite [10, 12].

Variating the summed action $S_{\mathrm{E}}+S_{\text {matter }}$ with respect to $P(r)$ and $N(r)$, the two Einstein equations are given by

$$
\begin{aligned}
& N^{\prime}=\frac{1-N}{r}-8 \pi G r\left(\mathcal{U}+N \mathcal{K}+\frac{\mathcal{K}_{0}}{P^{2}}+\frac{\mathcal{U}_{0}}{P^{2} N}\right), \\
& P^{\prime}=8 \pi G r\left(P \mathcal{K}+\frac{\mathcal{U}_{0}}{P N^{2}}\right) .
\end{aligned}
$$

Variating the action with respect to the matter fields $f(r), \rho(r), w(r)$ and $y(r)$, one has the following four matter equations of motion

$$
\begin{aligned}
\left(P N f^{\prime}\right)^{\prime} & =P\left[\frac{f\left(f^{2}-1\right)}{r^{2}}+\frac{g^{2}}{4} v^{2} f \rho^{2}\right]-\frac{v^{2} f w^{2}}{P N}, \\
\left(r^{2} P N \rho^{\prime}\right)^{\prime} & =\frac{1}{2} P \rho f^{2}+\frac{\lambda v^{2}}{2} r^{2} P \rho\left(\rho^{2}-1\right)-\frac{v^{2}}{4 P N} r^{2} \rho(w-y)^{2}, \\
\left(r^{2} P^{-1} w^{\prime}\right)^{\prime} & =\frac{2}{P N} f^{2} w+\frac{g^{2} v^{2}}{4 P N} r^{2} \rho^{2}(w-y), \\
\left(r^{2} P^{-1} y^{\prime}\right)^{\prime} & =\frac{g_{Y}^{2} v^{2}}{4 P N} r^{2} \rho^{2}(y-w) .
\end{aligned}
$$

\section{$3 \quad$ Hairy magnetic black holes}

\subsection{Masses and profiles}

For the magnetic black holes, existing papers have mainly studied the $\mathrm{SU}(2)$-gauge theory case $[6,7,9-12]$. Here, we focus on the $\mathrm{SM}$ electroweak $\mathrm{SU}(2)_{W} \times \mathrm{U}(1)_{Y}$ Lagrangian with our knowledge of the SM Higgs boson mass or the quartic coupling $\lambda[15,16]$. The equations of motion can be obtained from eqs. $(2.14)(2.15)(2.16)(2.17)$ by setting $w(r)=y(r)=0$. We are looking for solutions with the existence of a horizon $r_{H}$. Defining

$$
N(r)=1-\frac{2 G F(r)}{r}+\frac{4 \pi G}{g_{Y}^{2} r^{2}},
$$


we have the asymptotic mass of the system to be $M=F(\infty)$. Substituting eq. (2.15) with $P^{\prime} / P=8 \pi G r \mathcal{K}$ into eqs. $(2.16)(2.17)$, we have three equations for three fields $F(r)$ [or $N(r)$ via $(3.1)], f(r), \rho(r)$

$$
\begin{aligned}
F^{\prime} & =4 \pi r^{2}\left(\mathcal{U}_{1}+N \mathcal{K}\right), \\
\left(N f^{\prime}\right)^{\prime}+8 \pi G r N f^{\prime} \mathcal{K} & =\frac{f\left(f^{2}-1\right)}{r^{2}}+\frac{g^{2}}{4} v^{2} f \rho^{2}, \\
\left(r^{2} N \rho^{\prime}\right)^{\prime}+8 \pi G r^{3} N \rho^{\prime} \mathcal{K} & =\frac{1}{2} \rho f^{2}+\frac{\lambda v^{2}}{2} r^{2} \rho\left(\rho^{2}-1\right) .
\end{aligned}
$$

Noting that the $1 / r^{2}$ term introduced in (3.1) is to have the equation of motion for $F(r)$ contain $\mathcal{U}_{1}$ without the last $1 / r^{4}$ term in $\mathcal{U}$ [see (2.13)].

At the event horizon with $N\left(r_{H}\right)=0$, one has

$$
F\left(r_{H}\right)=\frac{r_{H}}{2 G}+\frac{2 \pi}{g_{Y}^{2} r_{H}} \geq \frac{\sqrt{4 \pi}}{\sqrt{G} g_{Y}}=c_{W} \frac{\sqrt{4 \pi}}{\sqrt{G} e} \equiv c_{W} M_{\mathrm{eBH}}^{\mathrm{RN}},
$$

with $c_{W} \equiv \cos \theta_{W}$ and the extremal RN black hole mass $M_{\mathrm{eBH}}^{\mathrm{RN}} \equiv \sqrt{4 \pi} M_{\mathrm{pl}} / e$. Using (3.1)(3.2), we obtain a boundary condition

$$
N^{\prime}=\frac{1}{r}-8 \pi G r \mathcal{U}, \quad \text { at } r=r_{H} .
$$

From (3.3)(3.4), one has two more mixed BC's that are

$$
\begin{aligned}
& N^{\prime} f^{\prime}=\frac{f\left(f^{2}-1\right)}{r^{2}}+\frac{g^{2}}{4} v^{2} f \rho^{2}, \quad \text { at } r=r_{H}, \\
& N^{\prime} \rho^{\prime}=\frac{1}{2} \frac{f^{2} \rho}{r^{2}}+\frac{\lambda v^{2}}{2} \rho\left(\rho^{2}-1\right), \quad \text { at } r=r_{H} .
\end{aligned}
$$

Together with the two BC's at infinity, $f(\infty)=0$ and $\rho(\infty)=1$, there are totally five BC's that are required for the equations in $(3.2)(3.3)(3.4)$.

Before we solve these equations, we first discuss the ordinary $\mathrm{RN}$ black hole solution, for which $f(r)=0$ and $\rho(r)=1$. Solving (3.2), one has a simple solution for $F(r)$ as

$$
F(r)=M-\frac{2 \pi}{g^{2} r}
$$

With the solution of $P(r)=1$ to have an asymptotically flat metric, this matches the RN metric

$$
P^{2}(r) N(r)=N(r)=1-\frac{2 G M}{r}+\frac{4 \pi G}{g^{2} r^{2}}+\frac{4 \pi G}{g_{Y}^{2} r^{2}}=1-\frac{2 G M}{r}+\frac{4 \pi G}{e^{2} r^{2}} .
$$

The outer horizon is at

$$
r_{H} \equiv r_{+}=M G+\sqrt{M^{2} G^{2}-4 \pi G / e^{2}}
$$

provided that $M \geq M_{\mathrm{eBH}}^{\mathrm{RN}}=\sqrt{4 \pi} M_{\mathrm{pl}} / e$. Inverting the above relation, one has

$$
M_{\mathrm{BH}}^{\mathrm{RN}}=\frac{r_{H}}{2 G}+\frac{2 \pi}{e^{2} r_{H}} .
$$

For the extremal case, one has $r_{H}^{\min }=r_{+}^{\min }=\sqrt{4 \pi} /\left(e M_{\mathrm{pl}}\right)$. 
Coming back to the hairy magnetic black holes, there exist constraints on the horizon $r_{H}$ if one makes a few plausible assumptions: $N^{\prime}\left(r_{H}\right) \geq 0, f(r)$ is a monotonically decreasing function outside the horizon, while $\rho(r)$ is a monotonically increasing function. The requirement of $N^{\prime}\left(r_{H}\right) \geq 0$ means $1 / r_{H}>8 \pi G r_{H} \mathcal{U}\left(r_{H}\right)$ from (3.6). For small $r_{H}$, one has $\mathcal{U}\left(r_{H}\right) \approx 1 /\left(2 g_{Y}^{2} r_{H}^{4}\right)$, so the lower bound on $r_{H}$ is

$$
r_{H} \geq r_{H}^{\min } \equiv \frac{\sqrt{4 \pi G}}{g_{Y}}=c_{W} \frac{\sqrt{4 \pi}}{e M_{\mathrm{pl}}},
$$

which is smaller than the extremal $\mathrm{RN}$ black hole horizon radius by a factor of $c_{W}$. Applying those assumptions to the BC's in (3.7)(3.8), one has

$$
\begin{aligned}
\lambda v^{2} r_{H}^{2}\left[1-\rho^{2}\left(r_{H}\right)\right] & <f^{2}\left(r_{H}\right)<1-\frac{g^{2} v^{2} r_{H}^{2}}{4} \rho^{2}\left(r_{H}\right), \\
1-\frac{f^{2}\left(r_{H}\right)}{\lambda v^{2} r_{H}^{2}} & <\rho^{2}\left(r_{H}\right)<\frac{4}{g^{2} v^{2} r_{H}^{2}}\left[1-f^{2}\left(r_{H}\right)\right] .
\end{aligned}
$$

For the SM with $g=e / s_{W} \approx 0.65$ and $\lambda=0.26$ such that $g^{2}<4 \lambda$ and using the fact that both $f\left(r_{H}\right)$ and $g\left(r_{H}\right)$ are within zero and one, the upper bound on $r_{H}$ is

$$
r_{H} \leq \frac{2}{g v}=\frac{1}{m_{W}} \approx 2.5 \times 10^{-3} \mathrm{fm},
$$

which is the characteristic radius of the monopole.

To calculate the mass of the hairy black holes, we integrate (3.2) to obtain

$$
F(r)=\int_{r_{H}}^{r} d r^{\prime} e^{-\mathbb{K}\left(r^{\prime}, r\right)} 4 \pi r^{\prime 2}\left[\mathcal{K}\left(r^{\prime}\right)+\mathcal{U}_{1}\left(r^{\prime}\right)+\frac{4 \pi G}{g_{Y}^{2} r^{\prime 2}} \mathcal{K}\left(r^{\prime}\right)\right]+e^{-\mathbb{K}\left(r_{H}, r\right)} F\left(r_{H}\right),
$$

where the new function is defined as

$$
\mathbb{K}\left(r^{\prime}, r\right) \equiv 8 \pi G \int_{r^{\prime}}^{r} d r^{\prime \prime} \mathcal{K}\left(r^{\prime \prime}\right) r^{\prime \prime}
$$

Given that $G v^{2} \ll 1$, the exponential, $e^{-\mathbb{K}\left(r^{\prime}, r\right)}=1+\mathcal{O}\left(G v^{2}\right)$. Ignoring the terms equal to or higher than $\mathcal{O}\left(G v^{2}\right)$, the mass of a hairy magnetic black hole has a simple formula

$$
\begin{aligned}
M_{\mathrm{hMBH}}=F(\infty) & =\int_{r_{H}}^{\infty} d r^{\prime} 4 \pi r^{\prime 2}\left[\mathcal{K}\left(r^{\prime}\right)+\mathcal{U}_{1}\left(r^{\prime}\right)\right]+F\left(r_{H}\right) \\
& =\int_{r_{H}}^{\infty} d r^{\prime} 4 \pi r^{\prime 2}\left[\mathcal{K}\left(r^{\prime}\right)+\mathcal{U}_{1}\left(r^{\prime}\right)\right]+\frac{r_{H}}{2 G}+\frac{2 \pi}{g_{Y}^{2} r_{H}} .
\end{aligned}
$$

The first integration term can be thought as the outside hair contribution to the total system mass. In the limit of $r_{H} \ll 1 / m_{W}$, the integration is dominated by the region with $r^{\prime} \sim 1 / m_{W}$. One can then obtain the leading contribution by using a flat metric with $N(r)=1$. Ignoring terms proportional to $G$, the equations of motion for $f(r)$ and $\rho(r)$ are simply

$$
\begin{gathered}
f^{\prime \prime}=\frac{f\left(f^{2}-1\right)}{r^{2}}+\frac{g^{2}}{4} v^{2} f \rho^{2}, \\
\left(r^{2} \rho^{\prime}\right)^{\prime}=\frac{1}{2} \rho f^{2}+\frac{\lambda v^{2}}{2} r^{2} \rho\left(\rho^{2}-1\right),
\end{gathered}
$$



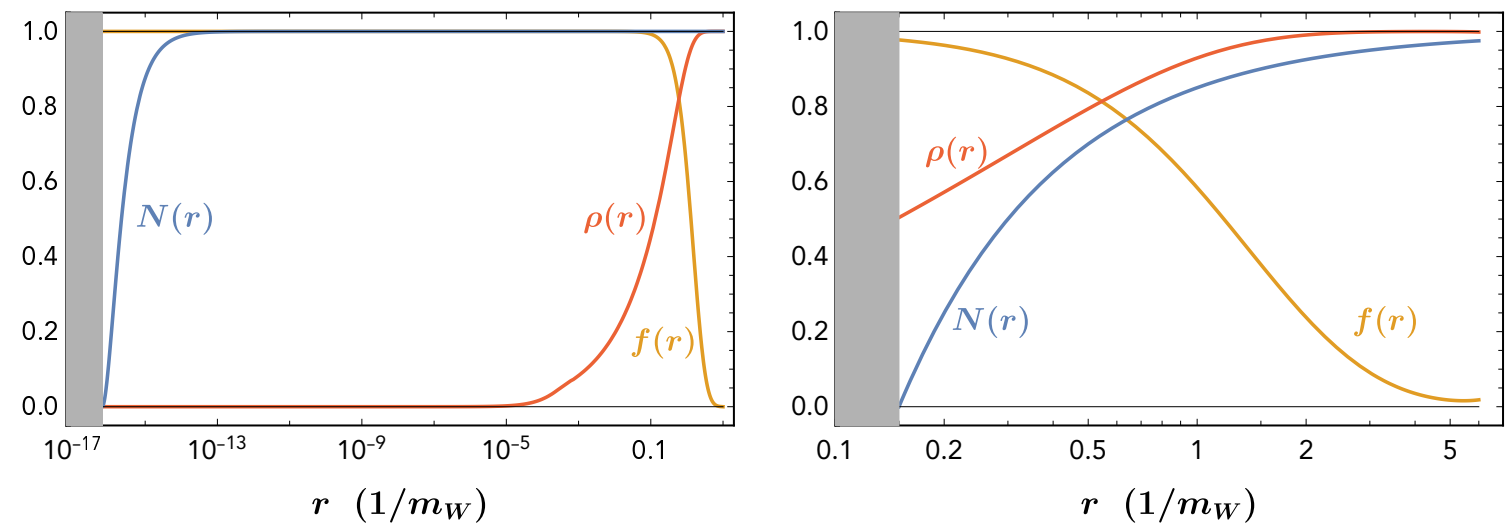

Figure 1. Profiles as a function of $r$ for the SM hairy magnetic black holes. Left: the horizon $r_{H}=c_{W} \sqrt{4 \pi} /\left(e M_{\mathrm{pl}}\right)$ for an extremal black hole. Right: a larger horizon with $r_{H}=0.15 / m_{W}$ with the Higgs vacuum expectation value (VEV) profile $\rho(r)$ to be half-restored at the horizon. Not shown here is the profile for $P(r)$, which is approximately one because of $P^{\prime} / P=\mathcal{O}\left(G v^{2}\right)$ and $P(\infty)=1$.

which are similar to the $\mathrm{t}$ 'hooft-Polyakov $\mathrm{SU}(2) / \mathrm{U}(1)$ magnetic monopole case with a different $W$ mass in terms of $v$. Numerically solving the differential equations and then calculating the first integration term in (3.19), the hairy magnetic black hole with $Q=2$ for $r_{H} \ll 1 / m_{W}$ has a mass

$$
\begin{aligned}
M_{\mathrm{hMBH}} & \approx \frac{r_{H}}{2 G}+\frac{2 \pi}{g_{Y}^{2} r_{H}}+0.75 \times \frac{4 \pi v}{g}=\frac{r_{H}}{2 G}+\frac{2 \pi c_{W}^{2}}{e^{2} r_{H}}+0.75 \times \frac{2 \pi v^{2}}{m_{W}} \\
& \geq c_{W} \frac{\sqrt{4 \pi} M_{\mathrm{pl}}}{e}+0.75 \times \frac{2 \pi v^{2}}{m_{W}}=\left(1.2 \times 10^{20}+3.6 \times 10^{3}\right) \mathrm{GeV} .
\end{aligned}
$$

Obviously, the hair part of the system contributes negligibly to the total mass. The upper mass of a $Q=2$ hairy magnetic black hole is

$$
M_{\mathrm{hMBH}} \leq M_{\mathrm{hMBH}}^{\max }=\frac{1}{2 G m_{W}}+\mathcal{O}\left(m_{W}\right) \approx 9.3 \times 10^{35} \mathrm{GeV} .
$$

Here, $m_{W}=g v / 2 \approx 80.4 \mathrm{GeV}$ is $\mathrm{W}$ gauge boson mass in the normal EW-broken vacuum.

Numerically solving the equations of motion with the BC's, we show two representative profiles in figure 1. In the left panel with $r_{H}=r_{H}^{\min }$ corresponding to the extremal case, the black hole sits well inside the hairy cloud. Around the event horizon, the Higgs VEV is very close to zero and the electroweak symmetry is almost completely restored. ${ }^{2}$ For potentially phenomenological applications, we also provide numerically fitted functions for both $f(r)$ and $\rho(r)$, that are good approximation for $r>0.1 m_{W}^{-1}$,

$$
\begin{aligned}
& f(r) \approx \frac{0.495 m_{W} r}{\sinh \left(1.1 m_{W} r\right)}+\frac{1.265 m_{W} r}{\sinh \left(2.3 m_{W} r\right)}, \\
& \rho(r) \approx\left(\operatorname{coth}\left[86\left(m_{W} r\right)^{1.2}\right]-\frac{1}{86\left(m_{W} r\right)^{1.2}}\right)\left(1-0.51 e^{-1.82 m_{W} r}\right) .
\end{aligned}
$$

\footnotetext{
${ }^{2}$ This is subject to corrections from the QCD condensation induced electroweak symmetry breaking.
} 


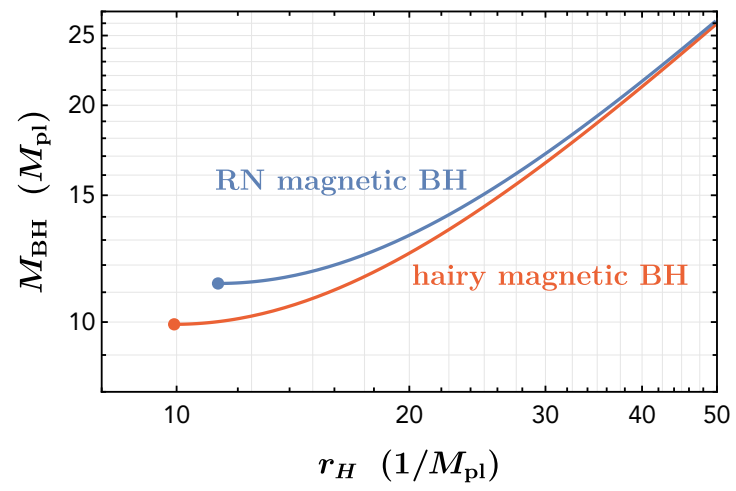

Figure 2. The black hole masses as functions of horizon radii for both the hairy and RN magnetic black holes. The left endpoints of the two curves correspond to the extremal black hole cases.

In the right panel of figure 1, we show an example of the non-extremal case with $r_{H}=$ $0.15 m_{W}^{-1}$ and the Higgs VEV half-restored at the event horizon. The total mass of system is well approximated by the term $r_{H} /(2 G)$ in eq. (3.19). The hair mass is $3.4 \mathrm{TeV}$ and smaller than the extremal case in (3.24). In figure 2, we show black hole masses as a function of horizon radii. For the same mass, the hairy magnetic black hole has a larger horizon radius, hence more entropy, than the $\mathrm{RN}$ magnetic black hole.

\subsection{Hawking temperature and black hole evaporation}

A non-extremal black hole evaporates via the Hawking radiation process. ${ }^{3}$ Based on the black hole thermodynamics, the black hole temperature is proportional to the surface gravity $\kappa$ at the horizon. For a Killing vector $\xi^{a}$, the surface gravity is $\kappa^{2}=-\left(\nabla^{a} \xi^{b}\right)\left(\nabla_{a} \xi_{b}\right) / 2$ evaluated at the horizon [26]. Using the metric in (2.4), we have

$$
T=\frac{\kappa}{2 \pi}=\frac{P\left(r_{H}\right) N^{\prime}\left(r_{H}\right)}{4 \pi} .
$$

The factor $P\left(r_{H}\right)=\exp \left[-\int_{r_{H}}^{\infty} d r 8 \pi G r \mathcal{K}(r)\right]=1+\mathcal{O}\left(G v^{2}\right)$. The other factor $N^{\prime}\left(r_{H}\right)$ can be obtained using (3.6): $N^{\prime}\left(r_{H}\right)=1 / r_{H}-8 \pi G r_{H} \mathcal{U}\left(r_{H}\right)=1 / r_{H}-4 \pi G /\left(g_{Y}^{2} r_{H}^{3}\right)+$ $\mathcal{O}\left(G v^{2} / r_{H}\right)$. So, we have

$$
T=\frac{1}{4 \pi r_{H}}\left(1-\frac{4 \pi G}{g_{Y}^{2} r_{H}^{2}}\right)=\frac{M_{\mathrm{pl}}^{2}}{2 \pi} \frac{\sqrt{M^{2}-M_{\mathrm{eBH}}^{2}}}{\left(M+\sqrt{M^{2}-M_{\mathrm{eBH}}^{2}}\right)^{2}},
$$

where the extremal hairy black hole mass is $M_{\mathrm{eBH}} \equiv c_{W} \sqrt{4 \pi} M_{\mathrm{pl}} / e$. The above temperature formula is similar to the $\mathrm{RN}$ black hole case, except that the extremal mass is smaller by a factor of $c_{W}$. This factor can be thought as the electroweak symmetry restoration near the event horizon, so the black hole carries magnetic hypercharge $2 \pi Q / g_{Y}=c_{W} 2 \pi Q / e$ with $Q=2$. Note that $T$ is not a monotonic function of $M$. For a very heavy mass $M \gg M_{\mathrm{eBH}}$, it

\footnotetext{
${ }^{3}$ If there exist monopoles in grand unified theories, the Schwinger discharge effect will evaporate away the magnetic charge of an hMBH. Based on SM interactions alone, there are no finite-energy monopoles, so the possibility of the Schwinger discharge effect is ruled out.
} 
is suppressed by $1 / M$ like the Schwarzschild case. For the opposite limit with $M \rightarrow M_{\mathrm{eBH}}$, $T \propto \sqrt{M-M_{\mathrm{eBH}}}$ and also suppressed. The maximal Hawking temperature has

$$
T^{\max }=\frac{e M_{\mathrm{pl}}}{12 \sqrt{3} \pi^{3 / 2} c_{W}} \approx 3.8 \times 10^{16} \mathrm{GeV},
$$

when $M=2 M_{\mathrm{eBH}} / \sqrt{3}$.

Depending on the early universe production mechanism, the initial state could be either a RN magnetic black hole or a hairy one. For the former case, the Hawking radiation leads to shrinking of $r_{H}$. When $r_{H}<1 / m_{W}$ or $M<M_{\mathrm{hMBH}}^{\max }$ in (3.25), the RN black hole is not classically stable and quickly converts to the hairy magnetic black hole [6]. At this moment, the temperature is around $6.4 \mathrm{GeV}$. The black hole horizon continues shrinking and $T$ keeps increasing. During this period, the Hawking radiation is dominated by the $2 d$ one (the ground state of charged fermions in the monopole background behaves as $2 d$ modes) with the radiated power as $[14,17]$

$$
P_{2}=-\frac{d M}{d t}=\frac{\pi g_{*}}{24} T^{2}\left(M, M_{\mathrm{eBH}}\right),
$$

with $g_{*}$ counts the number of left- and right-handed $2 d$ modes using the hypercharges of chiral fermions: $g_{*}=|Q|$ for $q_{L}, \ell_{L}, d_{R}, e_{R}$ and $g_{*}=2|Q|$ for $u_{R}$. Before the black hole mass becomes close to the extremal mass or in the limit of $M \gg M_{\mathrm{eBH}}$, it evaporates with a time scale of

$$
\tau_{\mathrm{I}} \approx \frac{512 \pi M^{3}}{g_{*} M_{\mathrm{pl}}^{4}}=\left(1.0 \times 10^{9} \mathrm{~s}\right) \times\left(M / M_{\mathrm{hMBH}}^{\max }\right)^{3},
$$

for $g_{*}=36$. So, for $M \gtrsim 10^{-3} M_{\mathrm{hMBH}}^{\max } \approx 10^{33} \mathrm{GeV}$, this period of evaporation is longer than the Big Bang nucleosynthesis (BBN) time $\mathcal{O}(1 \mathrm{~s})$. The black hole evaporation could leave observational imprints on the light-element abundance [27].

As the black hole approaches the extremal state, the Hawking temperature is approximately $T \simeq M_{\mathrm{pl}}^{2} \sqrt{M-M_{\mathrm{eBH}}} /\left(\sqrt{2} \pi M_{\mathrm{eBH}}^{3 / 2}\right)$. For $T \gtrsim m_{e}\left[M-M_{\mathrm{eBH}} \gtrsim 16 \pi^{7 / 2} c_{W}^{3} m_{e}^{2} /\right.$ $\left.\left(e^{3} M_{\mathrm{pl}}\right) \approx 4.1 \times 10^{-22} \mathrm{GeV}\right]$ or the lightest electrically-charged particle mass, $2 d$ radiation is still the dominant one. The evaporation time scale from some initial mass $M \simeq M_{\mathrm{eBH}}$ to the mass with $T \simeq m_{e}$ is estimated to be

$$
\tau_{\mathrm{II}}=\frac{384 \pi^{5 / 2} c_{W}^{3}}{e^{3} g_{*} M_{\mathrm{pl}}} \ln \left[\frac{M_{\mathrm{pl}}^{4}\left(M-M_{\mathrm{eBH}}\right)}{2 \pi^{2} m_{e}^{2} M_{\mathrm{eBH}}^{3}}\right] \approx 3.7 \times 10^{-37} \mathrm{~s},
$$

for $M=1.1 M_{\mathrm{eBH}}$ and $g_{*}=2$. This period of $2 d$ evaporation is very short.

As the black hole temperature continues to decrease till $T \lesssim m_{e}$, the $2 d$ evaporation is not active any more. The ordinary $4 d$ blackbody radiation has

$$
P_{4}=-\frac{d M}{d t} \approx \frac{\pi^{2} g_{*}}{120} 4 \pi R_{\mathrm{mono}}^{2} T^{4}\left(M, M_{\mathrm{eBH}}\right) .
$$

Here, $g_{*}=2$ for photon and $g_{*}=21 / 4$ for three chiral neutrinos; $R_{\text {mono }} \approx 1 / m_{W}$ is the monopole hair size, within which both photons and neutrinos are stored assuming that the 
hairy part is heated up to the Hawking temperature of the black hole. Solving the above equation, we have the black hole mass approaching the extremal state as

$$
M \approx M_{\mathrm{eBH}}+\frac{120 \pi M_{\mathrm{eBH}}^{6} m_{W}^{2}}{g_{*} M_{\mathrm{pl}}^{8}} \frac{1}{t} .
$$

During this long process, the Hawking temperature is

$$
T \approx \sqrt{\frac{60}{\pi g_{*}}} \frac{m_{W} M_{\mathrm{eBH}}^{3 / 2}}{M_{\mathrm{pl}}^{2}} t^{-1 / 2} \approx 2.7 \times 10^{-27} \mathrm{GeV} \times\left(\frac{t_{\mathrm{univ}}}{t}\right)^{1 / 2},
$$

with $g_{*}=2$ and the age of the Universe $t_{\text {univ }} \approx 4.35 \times 10^{17} \mathrm{~s}$. After the initial evaporation time $t_{\mathrm{I}}$ in (3.32), the Hawking temperature is already suppressed to be smaller than $\sim 10^{-23} \mathrm{GeV}$, which is tiny.

\section{Hairy dyonic black holes}

\subsection{Masses and profiles}

For the dyonic black hole case, we have six equations of motion in eqs. (2.14)-(2.19) with four second-order-differential and two first-order-differential equations. We need $2 \times 4+2=10$ BC's to solve the system. Four BC's are the mixed boundary conditions and can be obtained by simply evaluating (2.16)-(2.19) at $r=r_{H}$. Noting that $N\left(r_{H}\right)=0$ and to have a non-divergent BC's, two of these four BC's become simple conditions for $w(r)$ and $y(r)$ as $[28,29]$

$$
w\left(r_{H}\right)=0, \quad y\left(r_{H}\right)=0 .
$$

Two more BC's are the existence of the event horizon and the asymptotically flat spacetime at infinity and four more BC's are the profiles at infinity

$$
N\left(r_{H}\right)=0, \quad P(\infty)=1, \quad f(\infty)=0, \quad \rho(\infty)=1, \quad w(\infty)=y(\infty)=w_{\infty} .
$$

Noting from $(2.10)(2.11), w(\infty)=y(\infty)$ can assure vanishing $Z$ profile at infinity, but not the photon profile.

Similar to the magnetic case, we define the function $F(r)$ via (3.1), and (2.14) can be replaced by

$$
F^{\prime}=4 \pi r^{2}\left(\mathcal{U}_{1}+N \mathcal{K}+\frac{\mathcal{K}_{0}}{P^{2}}+\frac{\mathcal{U}_{0}}{P^{2} N}\right)
$$

The boundary condition $N\left(r_{H}\right)=0$ can be converted to

$$
F\left(r_{H}\right)=\frac{r_{H}}{2 G}+\frac{2 \pi}{g_{Y}^{2} r_{H}}
$$

which is similar to the magnetic case. At $r=r_{H}$, the derivative $N^{\prime}\left(r_{H}\right)$ is given by

$$
N^{\prime}\left(r_{H}\right)=\frac{1}{r_{H}}-\left.8 \pi G r_{H}\left(\mathcal{U}+\frac{\mathcal{K}_{0}}{P^{2}}+\frac{\mathcal{U}_{0}}{P^{2} N}\right)\right|_{r_{H}} .
$$


The EOMs admit the ordinary RN dyonic black hole solutions with $f(r)=0, \rho(r)=1$, $P(r)=1$ and $\omega(r)=y(r)$. For this solution, one has $\left(r^{2} y^{\prime}\right)^{\prime}=0$. Subject to the two BC's, $y\left(r_{H}\right)=0$ and $y(\infty)=y_{\infty}$, the solution is

$$
w(r)=y(r)=y_{\infty}\left(1-\frac{r_{H}}{r}\right) .
$$

From (2.10), the zeroth component of the photon field in the unitary gauge is

$$
A_{0}=-e v\left(\frac{1}{g^{2}}+\frac{1}{g_{Y}^{2}}\right) y(r)=-\frac{v}{e} y(r) .
$$

The asymptotic radial electric field is

$$
E_{r}=F_{0 r}=-\partial_{r} A_{0}=\frac{v}{e} y^{\prime}(r)=\frac{v r_{H} y_{\infty}}{e r^{2}} \equiv \frac{q e}{4 \pi r^{2}},
$$

from which, one can identify $y_{\infty}=q e^{2} /\left(4 \pi v r_{H}\right)$. The equation of motion for $F(r)$ becomes

$$
F^{\prime}=\frac{2 \pi}{g^{2} r^{2}}\left(1+\frac{g^{2}}{e^{2}} y_{\infty}^{2} v^{2} r_{H}^{2}\right)=\frac{2 \pi}{g^{2} r^{2}}\left[1+\frac{q^{2} g^{2} e^{2}}{(4 \pi)^{2}}\right]
$$

The solution to the above equation is

$$
F(r)=M-\frac{2 \pi}{g^{2} r}\left[1+\frac{q^{2} g^{2} e^{2}}{(4 \pi)^{2}}\right] .
$$

Substituting this into (3.1), we have the RN metric

$$
P^{2}(r) N(r)=N(r)=1-\frac{2 G M}{r}+\frac{4 \pi G}{e^{2} r^{2}}+\frac{G q^{2} e^{2}}{4 \pi r^{2}}=1-\frac{2 G M}{r}+G \frac{Q^{2} e_{\mathrm{M}}^{2}+q^{2} e^{2}}{4 \pi r^{2}},
$$

which matches the RN black hole with the magnetic and electric charge of $(Q=2, q)$ and the magnetic coupling of $e_{\mathrm{M}}=2 \pi / e$. The extremal dyonic RN black hole mass and horizon radius are

$$
M_{\mathrm{eBH}}^{\mathrm{RN}}=\frac{\sqrt{4 \pi} M_{\mathrm{pl}}}{e}\left[1+\frac{q^{2} e^{4}}{(4 \pi)^{2}}\right]^{1 / 2}, \quad r_{H}^{\min }=r_{+}^{\min }=\frac{\sqrt{4 \pi}}{e M_{\mathrm{pl}}}\left[1+\frac{q^{2} e^{4}}{(4 \pi)^{2}}\right]^{1 / 2} .
$$

Coming back to the hairy dyonic black holes, we first work out the constraints on $r_{H}$. The lower limit can be obtained by requiring $N^{\prime}\left(r_{H}\right) \geq 0$ from (2.14). In the limit of $G v^{2} \ll 1, P(r) \approx 1$ and $N(r) \approx\left(1-r_{H} / r\right)^{2}$ for the extremal case. In the limit of $r \rightarrow r_{H}$, one has $y(r) \propto r_{H}^{-1}\left(r-r_{H}\right), w(r) \propto m_{W} r_{H}^{-1}\left(r-r_{H}\right)^{2}, f(r) \approx 1$ and $\rho(r) \propto\left(1-r_{H} / r\right)^{(\sqrt{3}-1) / 2}$. The leading contribution to $\mathcal{K}_{0} \approx v^{2}\left[y^{\prime}\left(r_{H}\right)\right]^{2} /\left(2 g_{Y}^{2}\right)$ is $\mathcal{O}\left(1 / r_{H}^{2}\right)$ instead of $\mathcal{O}\left(1 / r_{H}^{4}\right)$ for the $\mathrm{RN}$ black hole case. Therefore, keeping the dominant $1 /\left(r_{H}^{4}\right)$ term in $\mathcal{U}$ for $(4.5)$ and in the limit of $G v^{2} \ll 1$, we have

$$
N^{\prime}\left(r_{H}\right) \approx \frac{1}{r_{H}}-8 \pi G r_{H}\left(\frac{1}{2 g_{Y}^{2} r_{H}^{4}}\right) \geq 0
$$


From the above condition, one has the hairy black hole horizon above the extremal one

$$
r_{H} \geq r_{H}^{\min }=\frac{\sqrt{4 \pi G}}{g_{Y}}=c_{W} \frac{\sqrt{4 \pi}}{e M_{\mathrm{pl}}},
$$

which is the same as the hairy magnetic black hole case. One may wonder why there is not any additional $q$-dependent correction, which is present for the extremal RN dyonic black hole case in (4.12). This can be understood by looking at the behaviors of (2.19) in $r$, since $r^{2} y^{\prime}$ is effectively the amount of hypercharge as a function of $r$. At large $r$, one has $y(r) \approx w(r), r^{2} y^{\prime}$ stays constant and the effective charge does not change much. For a smaller $r$ close to the scale of $r_{H}$ and because of the double zero in $N(r)$, the positive slope of the effective charge in $r$ becomes very large. As a result, the effective charge decreases dramatically as $r$ decreases. As $r$ is very close to $r_{H}$, the effective charge becomes approximately zero. So, the extremal hairy dyonic black hole has a magnetic black hole in the core and dyonic hair outside.

For the upper bound on $r_{H}$, noting that both $w(r) \propto\left(r-r_{H}\right)$ and $y(r) \propto\left(r-r_{H}\right)$ and $N \propto\left(r-r_{H}\right)$ when $r \rightarrow r_{H}$ for a non-extremal black hole, so the last terms in (2.16) and (2.17) can be ignored. Requiring $f^{\prime}\left(r_{H}\right) \leq 0$ and $\rho^{\prime}\left(r_{H}\right) \geq 0$, one can derive the same upper limit $r_{H} \leq 1 / m_{W}$ as in (3.16) for the hairy magnetic black hole case. Hence, one has a similar upper mass limit for hairy dyonic black holes $M_{\mathrm{hDBH}}^{\max } \approx 9.3 \times 10^{35} \mathrm{GeV}$ as in (3.25).

Based on (2.16) and requiring an exponentially decreasing behavior for $f(r)$ at large $r$, the electric charge is bounded from above [30]. The constraint on the large- $r$ value $y_{\infty}$ of $y(r)$ to be $y_{\infty} \leq g / 2$, which could be converted to an upper bound on the electric charge $q$. Although an analytic result can not be obtained, one could make some approximation to estimate the magnitude of the upper bound on $q$. To proceed, we make a step-function assumption for $f(r)$ such that $f(r)=1(0)$ for $r \leq(>) m_{W}^{-1}$. In the large $r$ limit and from (2.19) and (2.18), one has $y(r)=y_{\infty}-b_{1} / r$ and $w(r)=y_{\infty}-b_{2} / r$. Matching the electric charge, one has $b_{1} \sim b_{2} \sim b=q e^{2} /(4 \pi v)$. In the small $r$ limit and noting that $N(r) \approx\left(1-r_{H} / r\right)$ for non-extremal cases, one has $y(r) \propto r_{H}^{-1}\left(r-r_{H}\right)$ and $w(r)=c_{2}\left(r-r_{H}\right)$. Requiring both $w(r)$ and $w^{\prime}(r)$ to be continuous at $r=m_{W}^{-1}$, one has $b \approx m_{W}^{-1} y_{\infty} / 2$. The condition of $y_{\infty} \leq g / 2$ becomes $q \lesssim 2 \pi / e^{2}$, which says that the electric charge times the electric coupling can not be larger than the magnetic charge times the magnetic coupling to have a hairy dyonic black hole. There is no such requirement for the ordinary RN dyonic black holes.

In the limit of $G v^{2} \ll 1$ and integrating (4.3), we have the mass of a hairy dyonic black hole as

$$
\begin{aligned}
M_{\mathrm{hDBH}} & =F(\infty)=\int_{r_{H}}^{\infty} d r^{\prime} 4 \pi r^{\prime 2}\left[\mathcal{U}_{1}\left(r^{\prime}\right)+N \mathcal{K}\left(r^{\prime}\right)+\frac{\mathcal{K}_{0}\left(r^{\prime}\right)}{P^{2}}+\frac{\mathcal{U}_{0}\left(r^{\prime}\right)}{P^{2} N}\right]+F\left(r_{H}\right) \\
& \approx \int_{\sim 10 r_{H}}^{\infty} d r^{\prime} 4 \pi r^{\prime 2}\left[\mathcal{U}_{1}\left(r^{\prime}\right)+\mathcal{K}\left(r^{\prime}\right)+\mathcal{K}_{0}\left(r^{\prime}\right)+\mathcal{U}_{0}\left(r^{\prime}\right)\right]+\frac{r_{H}}{2 G}+\frac{2 \pi}{g_{Y}^{2} r_{H}},
\end{aligned}
$$

where in the second line the hair mass can be calculated by treating the spacetime as a flat one. In the limit of $r_{H} \ll m_{W}^{-1}$, the equations of motion for $f(r), \rho(r), w(r)$ and $y(r)$ can be solved with $N(r) \approx 1$ and $P(r) \approx 1$. For a small electric charge with $q \ll 2 \pi / e^{2}$, 


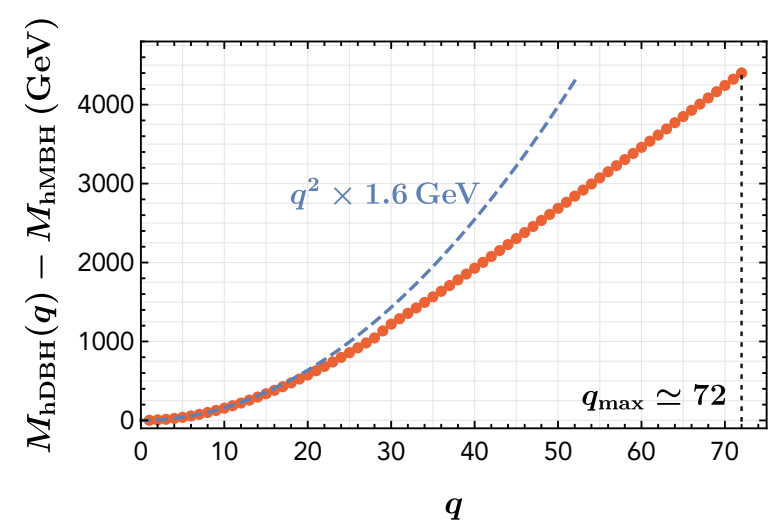

Figure 3. The mass difference between extremal hairy dyonic and magnetic black holes for different electric charge $q$. The upper charge limit $q_{\max } \simeq 72$ or $\mathcal{O}\left(2 \pi / e^{2}\right)$ is obtained by requiring an exponentially decreasing behavior for $f(r)$ at large $r$.

one can first ignore the last terms in (2.16) and (2.17) and obtain the solution for $f(r)$ and $\rho(r)$. Substituting them into (2.18) and (2.19), one can solve just two coupled differential equations to obtain $w(r)$ and $y(r)$. We have also solved the full-coupled equations to check that this approximation is good. For $q \ll 2 \pi / e^{2}$, a simple formula for the mass in terms of $q$ is given by

$$
\begin{aligned}
M_{\mathrm{hDBH}}(q) & \approx \frac{r_{H}}{2 G}+\frac{2 \pi c_{W}^{2}}{e^{2} r_{H}}+\left(0.75+0.00034 q^{2}\right) \times \frac{2 \pi v^{2}}{m_{W}} \\
& \approx M_{\mathrm{hMBH}}+q^{2} \times 1.6 \mathrm{GeV} .
\end{aligned}
$$

At the order-of-magnitude level, the mass difference between the dyonic and magnetic hairy parts is $M_{\mathrm{hDBH}}-M_{\mathrm{hMBH}}=\mathcal{O}\left(q^{2} \times \alpha m_{W}\right)$ with $\alpha$ as the fine-structure constant. Solving the fully coupled equations, in figure 3 we show the mass difference as a function of charges for the extremal black hole case. As discussed before, there exists an upper limit on the charge $q_{\max } \simeq 72$ (up to some numeric uncertainties) for the extremal case to have $w_{\infty}=y_{\infty}<g / 2$ or to have an exponentially decreasing behavior for $f(r)$ at large $r$. As one can see from this plot, the $q^{2}$ behavior matches very well the numerically calculated results for small $q$.

In the left panel of figure 4, we show the profiles $y(r)$ and $w(r)$ for the extremal hairy dyonic black hole with $q=1$ and $q=2$. For $y(r)$, it increases very fast from zero at $r=r_{H}$ to a plateau at $r \sim 10 r_{H}$. At $r \sim m_{W}^{-1}$, it continuously increases by a small amount into an asymptotic value $y_{\infty}$ at $r \gg m_{W}^{-1}$. For $w(r)$, it stays almost zero until $r \sim m_{W}^{-1}$, where it starts to increase to its asymptotic value $\omega_{\infty}=y_{\infty}$. For $r \gg m_{W}^{-1}$ and for $q \ll 2 \pi / e^{2}$, one has the following asymptotic behaviors

$$
\begin{aligned}
& w(r)=q \frac{e^{2} m_{W}}{4 \pi v}\left(5.2-\frac{1}{m_{W} r}-6.7 c_{W}^{2} \times \frac{e^{-m_{Z} r}}{m_{W} r}\right), \\
& y(r)=q \frac{e^{2} m_{W}}{4 \pi v}\left(5.2-\frac{1}{m_{W} r}+6.7 s_{W}^{2} \times \frac{e^{-m_{Z} r}}{m_{W} r}\right) .
\end{aligned}
$$



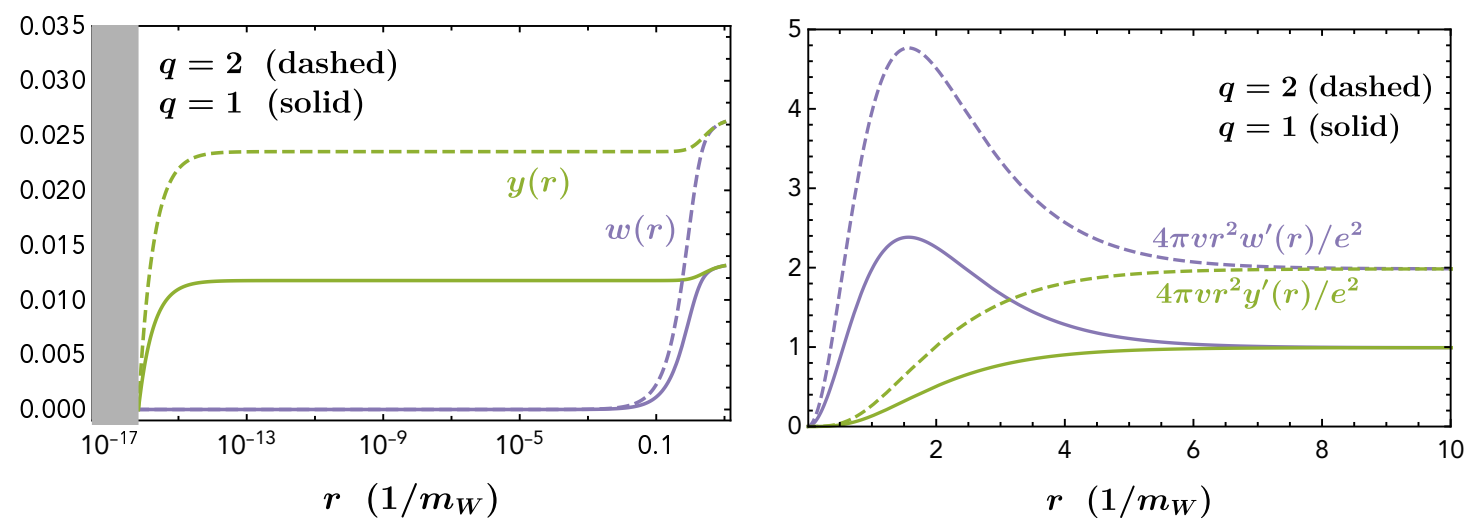

Figure 4. Left: $y(r)$ and $w(r)$ as a function of $r$ for the SM hairy dyonic black holes with an extremal black hole horizon $r_{H}=c_{W} \sqrt{4 \pi} /\left(e M_{\mathrm{pl}}\right)$. The profiles for other functions are approximately the same as in the left panel of figure 1. Right: the effective charges related to the two neutral gauge fields as a function of $r$.

Using (2.10) and (2.11), one can also obtain the asymptotic behaviors of the time components of photon and $Z$-boson fields and again in the small $q$ limit

$$
\begin{aligned}
& A_{0}(r) \stackrel{r \rightarrow \infty}{\longrightarrow}-q \frac{e m_{W}}{4 \pi}\left(5.2-\frac{1}{m_{W} r}\right), \\
& Z_{0}(r) \stackrel{r \rightarrow \infty}{\longrightarrow} 0.07 \times q \frac{e^{-m_{Z} r}}{r} .
\end{aligned}
$$

Because of the exponentially decreasing dependence for the time component of the $Z$-boson field, the hairy dyonic black hole has only a long-range electric field outside the hair radius of $\sim m_{Z}^{-1} \sim m_{W}^{-1}$.

\subsection{Some properties of hairy dyonic black holes}

Since the core region of a hairy dyonic black hole is only a magnetic black hole with electroweak symmetry restoration just outside the horizon. The black hole mass evolution as a function of time is the same as discussed in section 3.2. On the other hand, the existence of an electric charge for a dyonic black hole makes the hairy dyonic black holes different from the magnetic one. Here, we will focus on the extremal case with $r_{H}=r_{H}^{\min }$ in (4.14).

From figure 3, the mass difference between two hairy dyonic black holes with $\Delta q=1$ grows as $q$ increases. The largest difference is $M_{\mathrm{hDBH}}(72)-M_{\mathrm{hDBH}}(71) \approx 79.5 \mathrm{GeV}$, which is close to but below the free $W$-gauge boson mass. So, a charge- $q \mathrm{hDBH}$ can not decay into a charge- $q-1 \mathrm{hDBH}$ plus an on-shell $W$-gauge boson. On the other hand, it can be discharged by generating light charged fermions based on the charged current in the SM. Such processes have been studied and calculated based on the Julia-Zee dyons in the large charge limit [31-33]. For the $q=1$ case, it will decay into a hairy magnetic black hole plus kinematically-accessible charged particles in the SM

$$
\mathrm{hDBH}{ }^{-} \rightarrow \mathrm{hMBH}+\left\{\pi^{-}, \pi^{-} \pi^{0}, \pi^{-} \pi^{-} \pi^{+}, \mu^{-} \bar{\nu}_{\mu}, e^{-} \bar{\nu}_{e}, \cdots\right\}
$$

which is similar to the $\tau$-lepton decays since the mass difference is around $1.6 \mathrm{GeV}$ and close to the $\tau$-lepton mass. Different from the $\tau$ lepton, the dyon decay width is not suppressed 
by two powers of the Fermi constant. Based on a classical field theory calculation, its decay width is estimated to be [34]

$$
\Gamma\left(\mathrm{hDBH}^{-}\right) \sim \frac{\alpha}{4 \pi} m_{W}
$$

So, an hDBH decays very fast into an hMBH state on the time scale of $10^{-23} \mathrm{~s}$.

For a non-integer-charged $\mathrm{hDBH}$, the discharge processes can reduce its electric charge to be below one. After that, the hDBH with $q<1$ is also a cosmologically stable state. However, just based on the SM with all color-singlet states having integer charges, it is unlikely to form a non-integer-charged hDBH. One exception is from a non-zero CPviolating $\theta$-term, $\theta \alpha /(8 \pi) F_{\mu \nu} \widetilde{F}^{\mu \nu}$, in the SM Lagrangian. There is no zero-charged hMBH because of the $\theta$-dependent quantization condition [35]. The electric charge of $\mathrm{hDBH}$ is $n-\theta /(2 \pi)$ with $n$ as an integer. For $n=0$, the irrational-charged $\mathrm{hDBH}$ could be a cosmologically stable object. For $|\theta|>8 \pi^{2} m_{e}^{2} /\left(e^{2} m_{W}^{2}\right) \approx 3.2 \times 10^{-8}$, the electric field at the monopole radius $\sim m_{W}^{-1}$ is above $m_{e}^{2} / e$, so additional electron and positron clouds should also exist as additional hair for the black hole [33].

\section{Discussion and conclusions}

In this paper, we have performed a detailed calculation for the magnetic and dyonic black holes with electroweak hair based on the known SM interactions and general relativity. Only spherically symmetric black holes with a magnetic charge $Q=2$ have been considered in this paper. One could extend our calculation to non-spherical larger- $Q$ black holes (for the magnetic monopole states with a large $Q$, see $[36,37]$ for instance). A magnetic RN black hole will evolve into an hMBH if its horizon size $r_{H}<m_{W}^{-1}$, which sets an upper bound on the hMBH mass around $9.3 \times 10^{35} \mathrm{GeV}$. After subsequent Hawking radiation, it evolves into a (nearly) extremal hMBH. The total mass of an extremal hMBH with $Q=2$ is dominated by its core black hole mass $1.2 \times 10^{20} \mathrm{GeV}$, while its hair mass is only $3.6 \times 10^{3} \mathrm{GeV}$. The mass ratio $\left(\sim 10^{-17}\right)$ of the black hole hair over the total mass is much smaller than the ratio of a single strand of human hair weight over the total body weight $\left(\sim 10^{-12}\right)$ (this ratio is close to the ratio of a single red-blood cell over the total body weight). Although the black hole hair weight is small, it extends to a much larger region than the core black hole horizon size. Because of the electroweak symmetry restoration and effective $2 d$ massless modes for charged fermions, the $2 d$ Hawking radiation is much faster than the ordinary $4 d$ case.

We have only considered the tree-level Higgs potential with the value of $\lambda(\mu) \approx 0.26$ that gives $m_{h} \approx 125 \mathrm{GeV}$ in the EW broken vacuum and should be treated as a coupling evaluated at around the Higgs boson mass or $\mu=m_{h}$. The renormalization-group (RG) running effect of $\lambda(\mu)$ can be taken into account by considering $\lambda(\mu=1 / r)$. First, the running of $\lambda$ is very slow until $r$ reaches a location very close to the event horizon $r_{H}$. When $r \rightarrow r_{H}, \lambda$ could be very small and even become negative (assuming only SM physics). As can be seen from eq. (2.17), the term containing the quartic coupling $\lambda$ is suppressed at small $r$. So, the uncertainty from RG running effects are very suppressed. We have also neglected the RG running of the gauge couplings because their RG running is much slower. 
Here, we have only considered the electroweak sector of the SM to determine the properties of $\mathrm{hMBH}$ and $\mathrm{hDBH}$, and neglected the QCD sector of SM. We expect a QCD corona with a radius of $R_{\mathrm{QCD}} \sim 1 / \Lambda_{\mathrm{QCD}}$. Since in this region $e B>\Lambda_{\mathrm{QCD}}^{2}$, QCD is in a different phase from the normal vacuum phase without a magnetic field $[38,39]$. The QCD hair has a much smaller mass $\left(\sim \Lambda_{\mathrm{QCD}}\right)$ compared to the EW hair $(\sim 4 \pi v / g)$. So the effects of QCD hair on the black hole mass and Hawking temperature can be neglected. On the other hand, the QCD hair can modify the scattering cross sections of neutral hadrons off the magnetic black Hole. For charged particles, the long-range electromagnetic interaction provides the dominant scattering process [40].

We have only considered spherically symmetric metric and ansatz for Higgs and gauge fields. One could extend our study to a rotating hMBH. In this case, the starting point of this calculation is to employ Newman-Janis algorithm [41] for our spherically symmetric metric in eq. (2.4), in addition to that one also needs to consider axi-symmetric ansatz [42] for Higgs and gauge fields. One would obtain a system of coupled partial differential equations which would need to be solved to obtain field profiles. During its primordial evolution such a BH will spin down due to the Hawking radiation [43] and/or Schwinger pair production of electrically-charged particles. The latter one is in analogy with KerrNewman BH where a magnetically-charged BH with a non-zero angular momentum gives rise to an electric field [44]. If the induced electric field is larger than $m_{e}^{2}$ at the event horizon, charged particles can then be Schwinger pair produced and reduce the angular momentum of $\mathrm{BH}$. This effect can impose a bound on the angular momentum $a \equiv J / M$, where $J$ is the angular momentum and $M$ is the total mass, with $a<m_{e}^{2} M_{\mathrm{pl}}^{-3}$. With this bound on $a$, the modification on the extremal $\mathrm{BH}$ horizon radius and mass is at the order of $m_{e}^{4} / M_{\mathrm{pl}}^{4}$ and thus negligible.

Hairy magnetic black holes can be probed using a variety of astrophysical or directdetection methods. Motion of hMBHs in the galactic magnetic fields lead to the Parker bound on abundance of MBHs: $f_{*}<4 \times 10^{-4}$, where $f_{*}$ is the MBH abundance as the total fraction of dark matter abudance $[17,45]$. Similar to the magnetic monopole, MBHs can also be probed by direct-detection searches through MACRO and ancient Mica [17]. If one considers MBHs with higher magnetic charges with higher mass and larger event horizon radius, such objects could be probed by their annihilation signatures after they are captured by astrophysical objects [17], and binary mergers giving rise to gravitational waves along with the electromagnetic counterpart [45]. Also, the shadow of MBHs can be used to measure the value of the magnetic charge [46].

For an extremal hairy dyonic black hole, we have found that its core black hole is just a magnetic one without an electric charge, at least at the leading order in $G v^{2}$. Although this is different from the ordinary $\mathrm{RN}$ black hole case, it can be understood as the large cost of electrostatic energy at the limit of $r \rightarrow r_{H}$. An integer-charged hDBH can decay very quickly into a lower-charged $\mathrm{hDBH}$ and eventually into an $\mathrm{hMBH}$. Therefore, we don't anticipate a stable hDBH existing in the current universe. For a small electric charge $q \ll 2 \pi / e^{2}$, the dyonic hair mass is approximately $q^{2} \times 1.6 \mathrm{GeV}$ on top of $3.6 \times 10^{3} \mathrm{GeV}$ for the magnetic hair mass. So, a charge-one $\mathrm{hDBH}$ could be produced by cosmic rays hitting 
a stable hMBH via the processes like

$$
\begin{gathered}
e^{-}+\mathrm{hMBH} \rightarrow \mathrm{hDBH}^{-}+\nu_{e}, \\
p+\mathrm{hMBH} \rightarrow \mathrm{hDBH}^{+}+n,
\end{gathered}
$$

with the threshold energy of around $1.6 \mathrm{GeV}$ and the subsequent decays of an $\mathrm{hDBH}$ into other cosmic rays. The strength of the signature depends on the populations of hMBHs in the Universe.

Another interesting avenue beyond our paper is to study the early-universe productions of hairy magnetic black holes. The formation mechanism could be similar to ref. [27] with additional ingredients to form the monopole configuration based on the SM electroweak sector. As discussed around (3.32), if the initial hMBH is formed as a heavy non-extremal state with a mass above $\sim 10^{33} \mathrm{GeV}$, its early-stage evaporation time is long enough to leave imprints in the BBN. As also discussed in refs. [27, 45, 47, 48], a binary system with opposite-charged hMBHs could also be formed during the primordial formation or later structure formation periods. During the merging phase, the hairy dyonic black holes could be generated and its subsequent decays could provide temporal signatures. The final product contains a non-extremal black hole with bright Hawking radiation products that could be searched for by various telescopes.

It is entertaining to observe that the SM plus general relativity provides us exotic and cosmologically stable states. Along this general direction, one could also study the possibilities of gravitating states without a black hole, like the non-topological soliton stars by ref. [49], but with the known SM interactions.

\section{Acknowledgments}

We would like to thank Joshua Berger and Nicholas Orlofsky for useful discussion. The work is supported by the U.S. Department of Energy under the contract DE-SC-0017647. The work is in part supported by the Ray MacDonald Intra-mural Award.

Open Access. This article is distributed under the terms of the Creative Commons Attribution License (CC-BY 4.0), which permits any use, distribution and reproduction in any medium, provided the original author(s) and source are credited.

\section{References}

[1] C.W. Misner, K. Thorne and J. Wheeler, Gravitation, W.H. Freeman, San Francisco (1973) [INSPIRE].

[2] S. Droz, M. Heusler and N. Straumann, New black hole solutions with hair, Phys. Lett. B 268 (1991) 371 [INSPIRE].

[3] M. Heusler, S. Droz and N. Straumann, Stability analysis of selfgravitating skyrmions, Phys. Lett. B 271 (1991) 61 [INSPIRE].

[4] P. Bizon and T. Chmaj, Gravitating skyrmions, Phys. Lett. B 297 (1992) 55 [InSPIRE]. 
[5] G. Dvali and A. Gußmann, Skyrmion Black Hole Hair: Conservation of Baryon Number by Black Holes and Observable Manifestations, Nucl. Phys. B 913 (2016) 1001 [arXiv: 1605.00543] [INSPIRE].

[6] K.-M. Lee, V.P. Nair and E.J. Weinberg, A Classical instability of Reissner-Nordstrom solutions and the fate of magnetically charged black holes, Phys. Rev. Lett. 68 (1992) 1100 [hep-th/9111045] [INSPIRE].

[7] K.-M. Lee, V.P. Nair and E.J. Weinberg, Black holes in magnetic monopoles, Phys. Rev. D 45 (1992) 2751 [hep-th/9112008] [INSPIRE].

[8] B.R. Greene, S.D. Mathur and C.M. O'Neill, Eluding the no hair conjecture: Black holes in spontaneously broken gauge theories, Phys. Rev. D 47 (1993) 2242 [hep-th/9211007] [INSPIRE].

[9] K.-I. Maeda, T. Tachizawa, T. Torii and T. Maki, Stability of nonAbelian black holes and catastrophe theory, Phys. Rev. Lett. 72 (1994) 450 [gr-qc/9310015] [INSPIRE].

[10] K.-M. Lee and E.J. Weinberg, Nontopological magnetic monopoles and new magnetically charged black holes, Phys. Rev. Lett. 73 (1994) 1203 [hep-th/9406021] [INSPIRE].

[11] T. Tachizawa, K.-I. Maeda and T. Torii, NonAbelian black holes and catastrophe theory. 2. Charged type, Phys. Rev. D 51 (1995) 4054 [gr-qc/9410016] [inSPIRE].

[12] E.J. Weinberg, Magnetically charged black holes with hair, in 13th Symposium on Theoretical Physics: Field Theory and Mathematical Physics, (1994) [gr-qc/9503032] [INSPIRE].

[13] V. Cardoso and L. Gualtieri, Testing the black hole 'no-hair' hypothesis, Class. Quant. Grav. 33 (2016) 174001 [arXiv:1607.03133] [INSPIRE].

[14] J. Maldacena, Comments on magnetic black holes, arXiv:2004.06084 [INSPIRE].

[15] CMS collaboration, Observation of a New Boson at a Mass of $125 \mathrm{GeV}$ with the CMS Experiment at the LHC, Phys. Lett. B $\mathbf{7 1 6}$ (2012) 30 [arXiv:1207.7235] [INSPIRE].

[16] ATLAS collaboration, Observation of a new particle in the search for the Standard Model Higgs boson with the ATLAS detector at the LHC, Phys. Lett. B 716 (2012) 1 [arXiv: 1207.7214] [INSPIRE].

[17] Y. Bai, J. Berger, M. Korwar and N. Orlofsky, Phenomenology of magnetic black holes with electroweak-symmetric coronas, JHEP 10 (2020) 210 [arXiv:2007.03703] [INSPIRE].

[18] Y.M. Cho and D. Maison, Monopoles in Weinberg-Salam model, Phys. Lett. B 391 (1997) 360 [hep-th/9601028] [INSPIRE].

[19] J. Ellis, N.E. Mavromatos and T. You, The Price of an Electroweak Monopole, Phys. Lett. B 756 (2016) 29 [arXiv: 1602.01745] [INSPIRE].

[20] G. 't Hooft, Magnetic Monopoles in Unified Gauge Theories, Nucl. Phys. B 79 (1974) 276 [INSPIRE].

[21] A.M. Polyakov, Particle Spectrum in the Quantum Field Theory, JETP Lett. 20 (1974) 194 [INSPIRE].

[22] H. Georgi and S.L. Glashow, Unity of All Elementary Particle Forces, Phys. Rev. Lett. 32 (1974) 438 [INSPIRE].

[23] ATLAS and CMS collaborations, Combined Measurement of the Higgs Boson Mass in pp Collisions at $\sqrt{s}=7$ and $8 \mathrm{TeV}$ with the ATLAS and CMS Experiments, Phys. Rev. Lett. 114 (2015) 191803 [arXiv:1503.07589] [INSPIRE]. 
[24] P. Van Nieuwenhuizen, D. Wilkinson and M.J. Perry, On a Regular Solution of 't Hooft's Magnetic Monopole Model in Curved Space, Phys. Rev. D 13 (1976) 778 [inSPIRE].

[25] Y.M. Cho, K. Kimm and J.H. Yoon, Gravitationally Coupled Electroweak Monopole, Phys. Lett. B 761 (2016) 203 [arXiv:1605.08129] [INSPIRE].

[26] R.M. Wald, General relativity, Chicago University Press, Chicago, IL (1984) [DOI] [InSPIRE].

[27] Y. Bai and N. Orlofsky, Primordial Extremal Black Holes as Dark Matter, Phys. Rev. D 101 (2020) 055006 [arXiv: 1906. 04858] [INSPIRE].

[28] Y. Brihaye, B. Hartmann and J. Kunz, Gravitating dyons and dyonic black holes, Phys. Lett. B 441 (1998) 77 [hep-th/9807169] [INSPIRE].

[29] Y. Brihaye, B. Hartmann, J. Kunz and N. Tell, Dyonic nonAbelian black holes, Phys. Rev. D 60 (1999) 104016 [hep-th/9904065] [INSPIRE].

[30] B. Julia and A. Zee, Poles with Both Magnetic and Electric Charges in Nonabelian Gauge Theory, Phys. Rev. D 11 (1975) 2227 [inSPIRE].

[31] A.S. Blaer, N.H. Christ and J.-F. Tang, Anomalous fermion production by a Julia-Zee Dyon, Phys. Rev. Lett. 47 (1981) 1364 [INSPIRE].

[32] A.S. Blaer, N.H. Christ and J.-F. Tang, Fermion Emission From a Julia-Zee Dyon, Phys. Rev. D 25 (1982) 2128 [INSPIRE].

[33] C.G. Callan Jr., Disappearing Dyons, Phys. Rev. D 25 (1982) 2141 [inSPIRE].

[34] H. Sonoda, Decay of a Dyon, Nucl. Phys. B 238 (1984) 259.

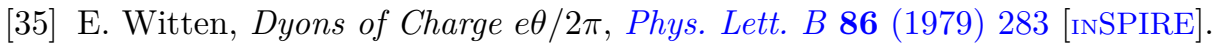

[36] R.S. Ward, A Yang-Mills Higgs Monopole of Charge 2, Commun. Math. Phys. 79 (1981) 317 [INSPIRE].

[37] C.J. Houghton and P.M. Sutcliffe, Tetrahedral and cubic monopoles, Commun. Math. Phys. 180 (1996) 343 [hep-th/9601146] [INSPIRE].

[38] I.A. Shovkovy, Magnetic Catalysis: A Review, Lect. Notes Phys. 871 (2013) 13 [arXiv: 1207.5081] [INSPIRE].

[39] D.E. Kharzeev, K. Landsteiner, A. Schmitt and H.-U. Yee, 'Strongly interacting matter in magnetic fields': an overview, Lect. Notes Phys. 871 (2013) 1 [arXiv:1211.6245] [InSPIRE].

[40] A.S. Goldhaber, Dirac Particle in a Magnetic Field: Symmetries and their Breaking by Monopole Singularities, Phys. Rev. D 16 (1977) 1815 [INSPIRE].

[41] E.T. Newman and A.I. Janis, Note on the Kerr spinning particle metric, J. Math. Phys. 6 (1965) 915 [INSPIRE].

[42] M. Kasuya, Exact Solution of a Rotating Dyon Black Hole, Phys. Rev. D 25 (1982) 995 [INSPIRE].

[43] D.N. Page, Particle Emission Rates from a Black Hole. 2. Massless Particles from a Rotating Hole, Phys. Rev. D 14 (1976) 3260 [inSPIRE].

[44] I. Semiz, Dyonic Kerr-Newman black holes, complex scalar field and cosmic censorship, Gen. Rel. Grav. 43 (2011) 833 [gr-qc/0508011] [inSPIRE].

[45] D. Ghosh, A. Thalapillil and F. Ullah, Astrophysical hints for magnetic black holes, Phys. Rev. D 103 (2021) 023006 [arXiv: 2009.03363] [INSPIRE]. 
[46] A. Allahyari, M. Khodadi, S. Vagnozzi and D.F. Mota, Magnetically charged black holes from non-linear electrodynamics and the Event Horizon Telescope, JCAP 02 (2020) 003 [arXiv: 1912.08231] [INSPIRE].

[47] L. Liu, O. Christiansen, Z.-K. Guo, R.-G. Cai and S.P. Kim, Gravitational and electromagnetic radiation from binary black holes with electric and magnetic charges: Circular orbits on a cone, Phys. Rev. D 102 (2020) 103520 [arXiv:2008.02326] [InSPIRE].

[48] L. Liu, O. Christiansen, Z.-K. Guo, R.-G. Cai and S.P. Kim, Gravitational and electromagnetic radiation from binary black holes with electric and magnetic charges: Elliptical orbits on a cone, arXiv:2011.13586 [INSPIRE].

[49] T.D. Lee and Y. Pang, Fermion Soliton Stars and Black Holes, Phys. Rev. D 35 (1987) 3678 [INSPIRE]. 